\title{
Thermo-refractive noise at high frequency: Beyond the conventional model
}

\author{
Nicolas Le Thomas, Ali Raza, Roel Baets \\ Photonics Research Group, INTEC Department, Ghent University-imec \\ Center for Nano- and Biophotonics, Ghent University, 9000 Gent, Belgium \\ Nicolas.LeThomas@UGent.be
}

\begin{abstract}
The detection limit of Raman sensors based on waveguides is restricted by a background that exhibits an unconventional exponential decay at high frequency. We discuss a new model that reveals the origin of this background. (C) 2018 The Author(s)

OCIS codes: (130.2790) Guided waves; (290.5860) Scattering, Raman; (230.7380) Waveguides, channeled
\end{abstract}

\section{Introduction}

Understanding noise issues is of paramount importance in waveguide-based Raman sensors as Raman scattering is an extremely weak inelastic scattering process: its conversion efficiency is around fifteen orders of magnitude smaller than the one of fluorescence. In particular, it has been recently observed that a background is present at high frequency in the optical spectrum of a single frequency laser beam having propagated through a silicon nitride (SiN) waveguide $[1,2]$.

Such a spectral background currently sets the ultimate lower bound on the detection limit of waveguide-based Raman sensors. Its origin has not been explained up to now. Here we propose a model that considers the fundamental thermodynamic fluctuations as the main contribution to the background. This model goes beyond the existing noise model [3] that is unable to reproduce the observed spectral shape of the background.

\section{Statement of the problem}

As schematically represented in Fig. 1a), when a single frequency laser beam of wavelength $\lambda_{0}$ propagates through a waveguide, its initial $\delta$ like optical spectrum $(\delta$ stands for the Dirac symbol) is modified by the appearance of a background that has a small amplitude, is spectrally large and decays exponentially at large frequency $\Omega$. For a spectrometer resolution of $\mathrm{d} v=9 \times 10^{10} \mathrm{~Hz}$ and an initial laser linewidth of $500 \mathrm{kHz}$, the amplitude of the background is $4 \times 10^{-9}$ smaller than the initial laser line amplitude in the case of a propagation through a $1 \mathrm{~cm}$ long silicon nitride waveguide of cross section $500 \mathrm{~nm} \times 200 \mathrm{~nm}$.
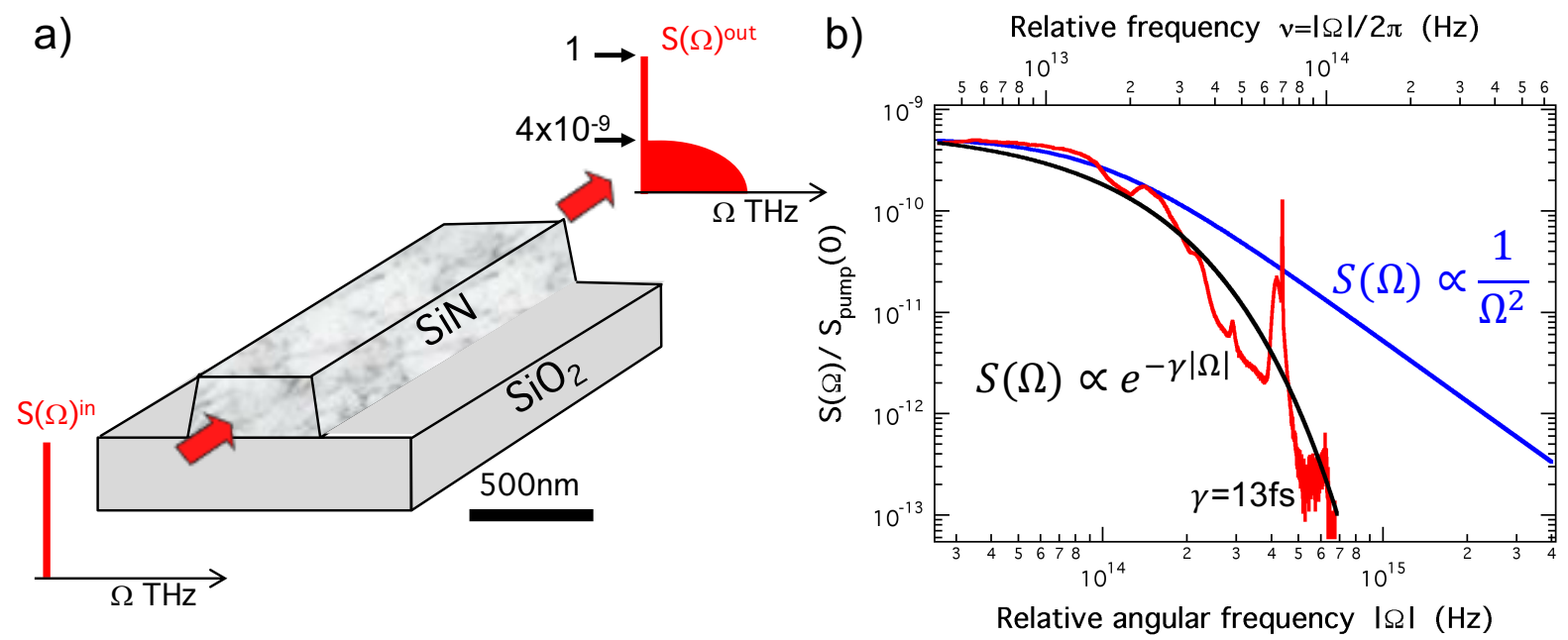

Fig.1. a) Schematic representation of the modification of the optical spectrum of a laser beam that propagates in a silicon nitride waveguide. b) Optical spectrum normalized according to optical spectrum of the input laser (pump); Red: Experimental spectrum, blue: Standard model, black: Current model with extended correlations. 
When the volume $\mathrm{V}$ of some medium at temperature $\mathrm{T}_{0}$ decreases, fundamental thermodynamic fluctuations start to play a role. In particular, a photonic structure of density $\rho$, specific heat $\mathrm{C}_{\mathrm{v}}$, and thermo-optic coefficient $\partial n / \partial T$ is subject to a thermo-refractive noise with the variance of the optical index fluctuations given by

$$
\left\langle\delta n^{2}\right\rangle=\left(\frac{\partial n}{\partial T}\right)^{2} \frac{k_{B} T_{0}}{\rho C_{V} \mathrm{~V}}
$$

where $\mathrm{k}_{\mathrm{B}}$ is the Boltzmann constant. The dynamics of the optical index fluctuations induces a phase noise on the light propagating in a waveguide, and consequently leads to a spectral noise contribution in the optical spectrum at the output of the waveguide. To model such a spectral noise contribution, the conventional approach makes use of the theory of thermal fluctuations [4] by assuming that the fluctuations at play are temporally and spatially $\delta$ correlated. As a result, a theoretical optical spectrum that decays as $1 / \Omega^{2}$ at high frequency is expected, which, however, does not match with the experimental optical spectrum (see Fig. 1b)).

\section{Main theoretical result}

It is possible to go beyond the conventional model of thermal fluctuations by considering that the local spontaneous heat fluxes that are generated by the constant motion of the elementary charges of the medium are temporally correlated with a characteristic time $\tau$. As a consequence, extended irreversible thermodynamics [5] predicts a spatial correlation of the fluctuation of the thermal field inside the medium. In particular, the spatial correlation length $\ell$ is given by $\ell=\sqrt{\tau D_{T}}$, where $D_{T}$ is the thermal diffusivity. At different times, the thermal field is subject to different statistical realization with all the same correlation length.

The fluctuations of the refractive index follow the same statistical properties as the one of the thermal field. The variance of these fluctuations is defined by equation (1) with the volume $\mathrm{V}$ replaced by the correlation volume $\ell^{3}$. The dynamics of the refractive index map can be described as the motion of elementary domains with a velocity distribution governed a generalized Boltzmann distribution [6]. This approach allows determining the time varying spectral density of the refractive index fluctuations and, consequently, the following optical spectrum of the light propagating in the waveguide:

$$
S(\Omega)=A_{0}^{2}\left\{\delta(\Omega)+4 \pi\left\langle\delta n^{2}\right\rangle \frac{L \ell}{\lambda_{0}^{2}} \frac{\ell^{2}}{\ell^{2}+2 W^{2}} \gamma e^{-\gamma|\Omega|}\right\},
$$

where $\gamma$ is a characteristic time close to $\frac{\hbar}{k_{B} T_{0}}$ and $\mathrm{W}$ is the radial half width of the transverse envelope of the intensity of the guided mode. The analytical formula (2) is the main result of our approach. It predicts the proper exponential shape of the experimental background (see Fig.1b)) in contrast to the conventional thermo-refractive noise model. It includes the intrinsic properties of the waveguide material, as well as the waveguide geometry. More specifically, it unveils that decreasing $\mathrm{W}$, i.e. decreasing the cross-section of the waveguide, increases the background. Besides, cancelling the correlation length minimizes the background.

Finally, the current approach is based on a hydrodynamic model, which does not take into account the possible crystalline nature of the medium. For instance, it cannot describe the spectral peak at a frequency of $v=70 \mathrm{THz}$ in Fig.1b). We anticipate that fabricating the waveguide with pure crystalline material should lead to a much smaller background.

\section{References}

[1] A. Dhakal, A.Z. Subramanian, P. Wuytens, F. Peyskens, N. Le Thomas, and Roel Baets, "Evanescent excitation and collection of spontaneous Raman spectra using silicon nitride nanophotonic waveguides,” Opt. Lett. 39, 4025-4028 (2014).

[2] S. A. Holmstrom, T. H. Stievater, D. A. Kozak, M. W. Pruessner, N. Tyndall, W. S. Rabinovich, R. A. McGill, J. B. Khurgin, “Trace gas Raman spectroscopy using functionalized waveguides," Optica 3, 891-896 (2016)

[3] S. Foster, A. Tikhomirov, M. Milnes, "Fundamental thermal noise in distributed feedback fiber lasers", IEEE J. Quantum Electron. 43, 378384 (2007).

[4] L. D. Landau, E. M. Lifshitz, Statistical Physics, part 1, $2^{\text {nd }}$ revised and enlarged edn (Pergamon Press, Oxford, 1980 ) §114.

[5] D. Jou, J. Casas-Vázquez, G. Lebon, Extended Irreversible Thermodynamics, $4^{\text {th }}$ edn (Springer 2010).

[6] N. Le Thomas, A. Dhakal, A. Raza, F. Peyskens, R. Baets, "Impact of fundamental thermodynamic fluctuations on light propagating in photonic waveguides made of amorphous materials," submitted (2017). 